ORIGINAL ARTICLE

\title{
Rofecoxib versus ibuprofen for acute treatment of migraine: a randomised placebo controlled trial
}

\author{
U K Misra, M Jose, J Kalita
}

Postgrad Med J 2004;80:720-723. doi: 10.1136/pgmj.2003.012393

See end of article for authors' affiliations

Correspondence to:

Professor Usha Kant Misra, Department of Neurology, Sanjay Gandhi PGIMS, Raebareily Road, Lucknow 226014, India; ukmisra@ sgpgi.ac.in

Submitted 9 July 2003

Accepted 28 October 2003
Background: Rofecoxib is a potent cyclo-oxygenase-2 inhibitor with a long duration of action. Its role in migraine has not been systematically evaluated.

Aim: To study the efficacy of rofecoxib in migraine.

Method: In a randomised placebo controlled trial rofecoxib $25 \mathrm{mg}$, ibuprofen $400 \mathrm{mg}$, and placebo were compared regarding their efficacy in relieving acute migraine attack. Migraine patients with 2-6 attacks per month were recruited. Headache severity, functional disability, and severity of associated symptoms were graded on a 0-3 scale. The primary endpoint was pain relief at two hours. Relief of associated symptoms and sustained pain relief for 24 hours were also noted.

Result: One hundred and twenty four patients were randomised into rofecoxib (42), ibuprofen (40), and placebo (42) groups. One hundred and one patients were followed up: 33 on rofecoxib, 35 ibuprofen, and 33 placebo. Patients' ages ranged from 16-62 (mean 31.4) years, and 83 were females. Pain relief at two hours was noted in $45.5 \%$ on rofecoxib, $55.6 \%$ on ibuprofen, and $9.1 \%$ in the placebo group. The associated symptoms at two hours were reduced in $39.4 \%$ on rofecoxib, $50 \%$ on ibuprofen, and $9.1 \%$ in the placebo group. Sustained 24 hour pain relief was noted in $36.4 \%$ on rofecoxib, $41 \%$ on ibuprofen, and $6.1 \%$ in the placebo group. In the ibuprofen group, five patients had abdominal pain but there were no side effects in those on rofecoxib or in the control group. Both rofecoxib and ibuprofen were significantly effective in relieving pain, associated symptoms at two hours, and in sustained pain relief. There was no significant difference between rofecoxib and ibuprofen in aborting acute migraine attacks. Conclusions: Both ibuprofen and rofecoxib were superior to placebo in aborting an acute migraine attack, and there was no significant difference in their efficacy in an acute migraine attack.
M igraine is one of the commonest causes of headache, and can be severe and last for hours to days. It is one of the commonest causes of absence from work and results in a considerable economic burden on society. Presently acetaminophen, non-steroidal anti-inflammatory drugs, ergot alkaloids, and triptans are used for treatment of an acute migraine attack. The choice of abortive treatment is based on its efficacy in relieving pain, lack of side effects, and cost. Rofecoxib is a selective cyclo-oxygenase (COX)-2 inhibitor with good gastrointestinal tolerance. In the treatment of arthritis rofecoxib was more effective in providing symptomatic relief of pain compared with placebo. ${ }^{1}$ Acetaminophen and celecoxib were similar in efficacy to ibuprofen, diclofenac, naproxen, and nabumetone. ${ }^{2}$ In the available literature, we could not find a randomised placebo controlled trial on the efficacy of rofecoxib in migraine. The present study, therefore, aims at evaluating the efficacy of rofecoxib and ibuprofen with placebo in the treatment of an acute attack of migraine.

\section{METHODS}

This randomised placebo controlled trial was conducted in a tertiary care teaching hospital between January 2001 and January 2002. The patients were recruited from the outpatient service of the neurology department. The protocol was approved by the institute's ethics committee and patients gave consent to participate in the trial and fill in the headache diary. The patients had at least a 12 month history of migraine with or without aura as defined by the criteria of the International Headache Society. ${ }^{3}$ All the patients were over 16 years of age and none had more than six attacks of migraine per month. The patients were randomised using random number tables into three groups: rofecoxib, ibuprofen, and placebo. The randomisation was done by one investigator (JK) and the responses were evaluated by another (MJ). The blinding was done by giving identical packets containing the medication; however, the tablets were not of identical appearance. The patients were asked to maintain a headache diary and instructed to note the headache severity, functional disability, and associated symptoms such as nausea, vomiting, photophobia, and phonophobia before and after the treatment. Severity of headache and functional disability were graded on a $0-3$ scale: grade $0=$ normal, grade $1=$ mild headache, grade $2=$ moderate headache, and grade $3=$ severe headache. Similarly functional disability was graded as $0=$ normal, $1=$ daily activities mildly impaired, $2=$ daily activities severely impaired, and $3=$ unable to perform daily activities, requiring bed rest. ${ }^{4}$ Patients with grade 3 functional disability needing bed rest during attacks and those with vomiting $20 \%$ or more of the time during a migraine attack were excluded. The severity of associated symptoms such as nausea, vomiting, photophobia, and phonophobia were also graded on a $0-3$ scale $(0=$ normal, $1=$ mild, $2=$ moderate, and 3 $=$ severe). A detailed clinical examination was carried out in all the patients. The patients were randomly prescribed rofecoxib $25 \mathrm{mg}$, ibuprofen $400 \mathrm{mg}$, or placebo (calcium $250 \mathrm{mg}$ ) kept in an identical looking packet. The patients were advised to take the study medication if headache was moderate or severe. Rescue medication (sumatriptan $100 \mathrm{mg}$ or piroxicam $20 \mathrm{mg}$ ) was advised only after two hours, if moderate or severe headache persisted (fig l).

Outcome was evaluated after one month or after two or more attacks, which were documented in the diary. The following three measures of efficacy were evaluated: (1) percentage of

Abbreviations: $\mathrm{Cl}$, confidence interval; $\mathrm{COX}$, cyclo-oxygenase; OR, odds ratio 


\begin{tabular}{|c|c|c|}
\hline & Registered/eligible patients $(n=124)$ & \\
\hline & Not randomised $(n=0)$ & \\
\hline & Reasons $(n=0)$ & \\
\hline \multirow[b]{2}{*}{ Rofecoxib } & Randomisation $(n=124)$ & \\
\hline & Ibuprofen & Placebo \\
\hline $\begin{array}{l}\text { standard intervention } \\
\text { ted }(n=42) \\
\text { eceive standard } \\
\text { on as allocated }(n=0)\end{array}$ & $\begin{array}{l}\text { Received standard intervention } \\
\text { as allocated }(n=40) \\
\text { Did not receive standard } \\
\text { intervention as allocated ( } n=0 \text { ) }\end{array}$ & $\begin{array}{l}\text { Received placebo as allocated } \\
(n=42) \\
\text { Did not receive placebo as } \\
\text { allocated }(n=0)\end{array}$ \\
\hline $\begin{array}{l}\text { up }(n=33) \\
\text { primary and } \\
\text { y outcome: } 2 \text { attacks } \\
\text { th }\end{array}$ & $\begin{array}{l}\text { Followed up }(\mathrm{n}=35) \\
\text { Timing of primary and } \\
\text { secondary outcome: } 2 \text { attacks } \\
\text { or } 1 \text { month }\end{array}$ & $\begin{array}{l}\text { Followed up }(\mathrm{n}=33) \\
\text { Timing of primary and } \\
\text { secondary outcome: } 2 \text { attacks } \\
\text { or } 1 \text { month }\end{array}$ \\
\hline $\begin{array}{l}\text { wn }(n=0) \\
\text { on ineffective }(n=18) \\
\text { low up }(n=9) \\
=0 \text { 0) }\end{array}$ & $\begin{array}{l}\text { Withdrawn }(n=1) \\
\text { Intervention ineffective }(n=16) \\
\text { Lost to follow up }(n=6) \\
\text { Other }(n=0)\end{array}$ & $\begin{array}{l}\text { Withdrawn }(n=0) \\
\text { Placbo ineffective }(n=30) \\
\text { Lost to follow up }(n=8) \\
\text { Other }(n=0)\end{array}$ \\
\hline d trial $(n=33)$ & Completed trial $(n=35)$ & Completed trial $(n=33)$ \\
\hline
\end{tabular}

patients with pain relief at two hours, (2) percentage of patients free of associated symptoms - that is, absence of nausea, vomiting, photophobia, or phonophobia, and (3) percentage of patients with 24 hours of sustained pain relief-that is, absence of recurrence of headache and lack of requirement of antimigraine medication for 24 hours. Headache recurrence was defined as the recurrence of moderate to severe headache within 24 hours of dosing in patients who initially had relief of pain two hours after medication. Any side effects of the drugs up to 24 hours after ingestion were also noted.

This study was designed as a non-inferiority trial expecting rofecoxib to be as good as ibuprofen and superior to placebo. In order to detect the difference between the two drugs alpha risk was taken as 5\%, critical difference in the drugs was taken as $10 \%$, and the power calculation was done according to the method described by Agresti. ${ }^{5}$ Sample size was based on the response rate. Pain relief at two hours was considered important (efficacy of the drug) and the standard deviation was taken as \pm 2 . Based on this criterion the sample size was calculated to be at least 30 in each group. The effect of treatment-that is, percentage of patients with 24 hour pain relief, percentage of patients free of associated symptoms, and percentage of patients with pain relief at 24 hours-was studied by logistic regression analysis. Comparison of two proportions at a time was done using the $\mathrm{Z}$ test of proportion.

\section{RESULTS}

One hundred and twenty four patients with migraine who fulfilled the inclusion criteria were recruited. Altogether 101 were followed up and 23 lost to follow up. The age of the patients ranged from 16-62 (mean 32.3) years and 83 were females. The monthly headache frequency ranged from two to six, and headache duration ranged from 4-36 hours. Sleep and analgesics were relieving factors in $98 \%$ patients. There was no baseline difference in the severity of headache in the three groups. Before medication, headache was severe in $82 \%$ patients. Associated symptoms such as nausea were present in all, vomiting in $89 \%$, and photophobia and phonophobia in
Figure 1 Profile of randomised controlled trial.
$99 \%$ of the patients. Vomiting in these patients, however, did not exceed $20 \%$ of the time of the migraine attack.

\section{Efficacy analysis}

In the 101 patients included in this study, active drugs more frequently aborted the migraine attack compared with placebo at two hours. In the rofecoxib group $45.5 \%$ of the patients were relieved of pain and $39.4 \%$ were free of associated symptoms at two hours. In the ibuprofen group $55.6 \%$ patients were relieved of pain and 50\% were free of associated symptoms, while in the placebo group it was $9.1 \%$ for both measures. In the rofecoxib group, sustained pain relief was present in $36.4 \%$ patients, in the ibuprofen group $41.7 \%$, and in control group only $6.1 \%$. Two hour pain relief was significantly superior in rofecoxib (odds ratio (OR) 8.33, $95 \%$ confidence interval (CI) 2.12 to 32.65 , power 0.83 ) and ibuprofen (OR 12.50, 95\% CI 3.22 to 48.55, power 0.82 ) compared with placebo. In relieving associated symptoms rofecoxib (OR 6.5, 95\% CI 1.64 to 25.76 , power 0.83 ) and ibuprofen (OR 10.0, 95\% CI 2.57 to 38.76, power 0.82 ) were significantly superior to placebo. Twenty four hour sustained pain relief was also significantly better in rofecoxib (OR 8.86, 95\% CI 1.79 to 43.71 , power 0.81 ) and ibuprofen (OR 11.07, 95\% CI 2.29 to 53.54 , power 0.81 ) compared with placebo. On comparing the efficacy of ibuprofen and rofecoxib by $\mathrm{Z}$ test there was no significant difference in their ability to relieve pain at two hours $(Z=0.84, p=0.20)$, relief of associated symptoms $(Z=0.88, p=0.19)$, and 24 hour pain relief $(\mathrm{Z}=0.45, \mathrm{p}=0.33)$. Rofecoxib and ibuprofen were significantly effective in relieving pain at two hours (rofecoxib $\mathrm{Z}$ 3.32, $\mathrm{p}=0.0005$; ibuprofen $\mathrm{Z}=4.09, \mathrm{p}=0.000002$ ); relief of associated symptoms (rofecoxib $\mathrm{Z}=2.87, \mathrm{p}=0.002$; ibuprofen $Z=3.69, p=0.0001)$; and 24 hour sustained pain relief (rofecoxib $Z=3.01, p=0.001$; ibuprofen $Z=3.42$, $\mathrm{p}=0.0003)$. The patients in the rofecoxib group did not experience any side effects whereas five patients in the ibuprofen group experienced abdominal pain, which was severe in one. The response to various drugs is shown in fig 2. 


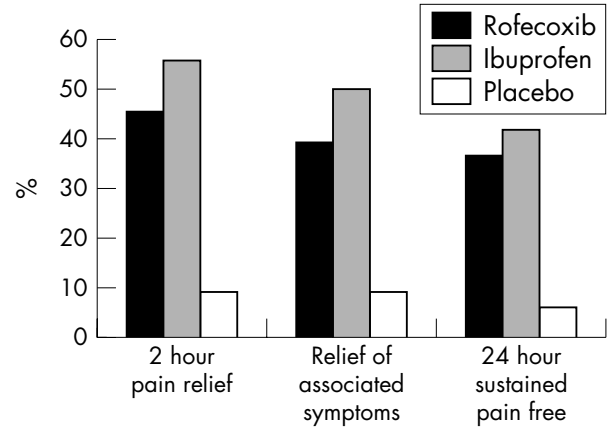

Figure 2 Efficacy of different treatment protocols in relieving pain at two hours, associated symptoms, and sustained 24 hour pain relief in acute migraine attack.

\section{DISCUSSION}

In this randomised placebo controlled trial both ibuprofen and rofecoxib were found to be effective compared with placebo in relieving acute migraine attacks. The efficacy of ibuprofen and rofecoxib was comparable. The dosage of $400 \mathrm{mg}$ ibuprofen and $25 \mathrm{mg}$ of rofecoxib have been decided on the basis of reported efficacy of these drugs in osteoarthritis. ${ }^{67}$ Ibuprofen in the dose of 400, 800, and $1200 \mathrm{mg}$ was proven to be more effective than placebo and comparable to $15 \mathrm{mg} / \mathrm{kg}$ paracetamol in children. ${ }^{7-9}$ Rofecoxib 12.5-25 mg daily was as effective as ibuprofen $800 \mathrm{mg}$ thrice daily for six weeks and diclofenac sodium $50 \mathrm{mg}$ thrice daily for 6-12 months in arthritis. ${ }^{6}$

The basis of efficacy and gastrointestinal safety of rofecoxib is attributed to its mechanism of action. Prostaglandins are formed by the interaction of COX-1 and COX-2. COX-1 is present mainly in gastric mucosa, kidney, and platelets. COX-2 is mainly an inducible form and present constitutively to some extent in the central nervous system, placenta, and the juxtaglomerular apparatus of the kidney. Both isoforms contribute to the inflammatory process but COX-2 is of special interest as it is induced by inflammation. Ibuprofen inhibits both the isoforms to a similar extent. COX-2 inhibitors are 100-1000 times more selective on COX-2 than COX-1, which is maintained even at a much higher dosage. ${ }^{10}$ Rofecoxib has been found to have a lower incidence of gastric ulcers, as evaluated by endoscopy, compared with ibuprofen ${ }^{11}$ and naproxen. ${ }^{12}$ However the gastrointestinal safety of rofecoxib has been questioned by some investigators. $^{13}$

The efficacy of COX-1 and COX-2 inhibitors in migraine may be due to an anti-inflammatory effect. In migraine, there is aseptic inflammation in the trigeminovascular system. ${ }^{14}{ }^{15}$ There is also release of vasoactive peptides-neurokinin A and calcitonin gene related peptides-that results in dilatation of vessels and leakage of albumin from blood vessels. None of these peptides is capable of disrupting the bloodbrain barrier. These neuropeptides also initiate the events resulting in formation of endothelial microvilli, endothelial vesicles, and vacuoles especially within the postcapillary dural venule. It also results in degranulation of mast cells, platelet aggregation, and leakage of plasma from meningeal vessels. ${ }^{16}$ Ibuprofen may inhibit the COX system, resulting in relief of pain. The other possible mechanisms may include its analgesic effect or more specific effect on the trigeminal and antinociceptive systems in the brainstem. ${ }^{17}{ }^{18}$

We have excluded patients with very frequent migraine attacks because they are not suitable for drug trials on an outpatient basis. Similarly patients with frequent vomiting exceeding $20 \%$ of the duration of an attack have been excluded because vomiting may interfere with drug absorption. In our study 22 patients were lost to follow up. The clinical characteristics - that is, age, sex, severity of pain, and associated symptoms - of the patients who were lost to follow up were not significantly different from the study group. There was no protocol deviation. The pain relief in our study was based on subjective evaluation by the patient, which is an inherent limitation of such studies. Our patients received identical drug packets, although the appearance of the drugs was not similar. This could have introduced a bias in a crossover study. We, however, did not follow a crossover design. While it is unlikely that the difference in tablets would create bias in a noncrossover study, it could be argued that patients at follow up could allude to the colour or size of a tablet, which effectively would unblind the observer. However, the observer was largely collating results from the patients' diaries; therefore, this source of potential bias is unlikely to be important.

The reported efficacy of ibuprofen in migraine is $42 \%-$ $72 \% .^{7919}{ }^{20}$ The response rate of ibuprofen in our study was $55.6 \%$. Ibuprofen is regarded as a relatively safe drug with good tolerance. ${ }^{7-9}{ }^{19}$ Only five patients in our study who were on ibuprofen therapy had pain in the abdomen; this was severe in one patient only. However, in the rofecoxib group, no side effects were noted, which is in agreement with the superior safety profile of rofecoxib. ${ }^{1021}$

The lower frequency of gastrointestinal side effects in the rofecoxib group is an advantage but in the ibuprofen group side effects were not serious. It seems logical to avoid ibuprofen in a patient with a history of acid peptic disease and in these patients, rofecoxib may be preferred over ibuprofen.

\section{ACKNOWLEDGEMENTS}

The authors thank Dr S K Mandal for statistical analysis and Rakesh Kumar Nigam for secretarial help.

\section{Authors' affiliations}

U K Misra, M Jose, J Kalita, Department of Neurology, Sanjay Gandhi Post Graduate Institute of Medical Sciences, Lucknow, India

\section{REFERENCES}

1 Hawkey CJ. COX-2 inhibitors. Lancet 1999;353:307-14.

2 Matheson AJ, Figgitt DP. Rofecoxib: a review of its use in the management of osteoarthritis, acute pain and rheumatoid arthritis. Drugs 2001;61:833-65.

3 Headache Classification Committee of the International Headache Society. A classification and diagnostic criteria for headache disorders, cranial neurolagia and facial pain. Cephalalgia 1988;8(57):1-96.

4 Adelman JU, Lipton RB, Ferrari MD, et al. Comparison of rizatriptan and other triptans on stringent measures of efficacy. Neurology 2001;57:1377-83.

5 Agresti A. Categorical data analysis. New York: John Wiley, 1990:239-40.

6 Caklwell J, Holt P. MK 0966, a specific COX 2 inhibitor, has clinical efficacy comparable to diclofenac in the treatment of knee and hip osteoarthritis (OA) in a 26 week control trial. Arthritis Rheum 1998;41:S83.

7 Hamalainen ML, Hoppu K, Valkeila E, et al. Ibuprofen or acetaminophen for the acute treatment of migraine in children: a double-blind, randomized, placebo-controlled, crossover study. Neurology 1997;48:103-7.

8 Havaka-Kanniainen $\mathrm{H}$. Treatment of acute migraine attack: ibuprofen and placebo compared. Headache 1989;29:507-9.

9 Kolster R, Nestvold K, Vilming ST. A double blind study of ibuprofen versus placebo in the treatment of acute migraine attacks. Cephalagia 1992; 12:169-71

10 Everts B, Wahrborg P, Kedner T. Cox 2 specific inhibitors - the emergence of new class of analgesic and antinflammatory drugs. Clin Rheum 2000;19:331-43

11 Laine L, Harper S, Simon T, et al. For the rofecoxib osteoarthritis endoscopy study group. A randomized trial comparing the effect of rofecoxib, a cyclooxygenase II-specific inhibitor, with that of ibuprofen on the gastrodoudenal mucosa of patients with osteoarthritis. Gastroenterology 1999; 117:776-83.

12 Hawkey CJ, Laine L, Simon T, on behalf of the Rofecoxib, Rheumatoid Arthritis Endoscopy Study Group, et al. Incidence of gastrodudenal ulcers in patients with rheumatoid arthritis after 12 weeks of rofecoxib, naproxen or placebo: a multicenter, randomized, double blind study. Gut 2003;52:820-6.

13 Juni P, Rutjes AWS, Dieppe PA. Are selective COX II inhibitors superior to traditional non-steroidal anti-inflammatory drugs? BMJ 2002;324:1287-9.

14 Welch NK, Cutrer FM, Goodsby PJ. Migraine pathogenesis: neural and vascular mechanisms. Neurology 2003;60:S9-14.

15 Moskowitz MA. Basic mechanisms in vascular headache. Neurol Clin 1990;8:801-15. 
16 Dimitriadou V, Buzzi MG, Theoharbes T, et al. Ultrastructural evidence for neurogenically mediated changes in blood vessels of the rat duramater and tongue following antidromic trigeminal stimulation. Neuroscience 1992:48:187-203.

17 Gobel H, Ernst M, Jeschke J, et al. Acetyl salisylic acid activates antinociceptive brainstem reflex activity in headache patients and healthy subjects. Pain 1992;48:187-95.

18 Kaube H, Hoskin KL, Goadsby PJ. Intravenous acetylsalisylic acid inhibits central trigeminal neurons in the dorsal horn of upper cervical spinal cord in the cat. Headache 1993;33:541-4.
19 Codispoti JR, Prior MJ, Fu M, et al. Efficacy of nonprescription doses of ibuprofen for treating migraine headache: a randomized controlled trial. Headache 2001;41:665-79.

20 Kellstein DE, Lipton RB, Geetha R, et al. Evaluation of a novel solubilized formulation of ibuprofen in the treatment of migraine headache: a randomized double blind, placebo-controlled, dose ranging study. Cephalalgia 2000;20:233-43.

21 Sang K, Fisher C, Mckay J, et al. MK-0966 a specific cox 2 inhibitor has clinical efficacy comparable to ibuprofen in the treatment of osteoarthritis (OA) in a 6 week control clinical trial. Arthritis Rheum 1998;41:S84.

\section{IMAGES IN MEDICINE}

\section{Flecainide challenge test for the diagnosis of Brugada syndrome}

A 52 year old white man presented having woken up feeling dizzy and unwell. He went to the bathroom and on returning to his bedroom he lost consciousness. He came around spontaneously with no evidence of neurological deficit. He did not have any palpitations. His initial electrocardiogram (ECG) showed $2 \mathrm{~mm}$ ST elevation in V2 only (fig 1). The ST elevation in the right side of the precordial leads was associated with a partial right bundle branch block pattern and varied spontaneously over time. There was no evidence of an acute coronary syndrome. We then performed an intravenous flecainide challenge. His baseline ECG showed minor concave ST elevation in V2 only but then he went on to develop classical Brugada changes with a maximum of $5 \mathrm{~mm}$ ST elevation in V2 associated with $\mathrm{T}$ wave inversion (fig 2). No arrhythmia was provoked. Coronary angiography was normal and he was fitted with an implantable cardiodefibrillator. Brugada syndrome is characterised by marked ST-segment elevation in the right precordial ECG leads (unrelated to ischaemia, electrolyte abnormalities, or structural heart disease) with a morphology of the QRS complex resembling a right bundle branch block, and is associated with a high risk for sudden death. ${ }^{1}$

The mechanisms responsible for the electrocardiographic actions of class I antiarrhythmic agents in Brugada syndrome are thought to be due to their ability to block sodium channels. ${ }^{2}$ Strong sodium channel blockade facilitates the loss of the right ventricular epicardial plateau phase by altering the balance of current at the end of phase 1 of the action potential from inward to outward. The result is an all or nothing repolarisation of the right ventricular action potential and marked abbreviation of the epicardial action potential duration. The loss of the plateau in right ventricular epicardium but not endocardium creates a transmural voltage gradient that manifests as an ST-segment elevation in the right precordial leads of the ECG.

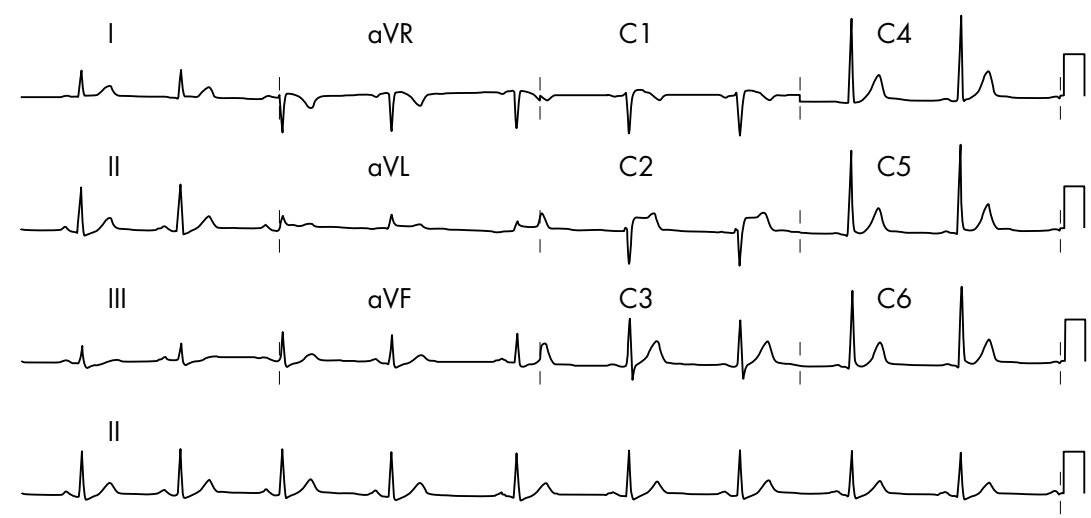

Figure 1 Initial ECG.

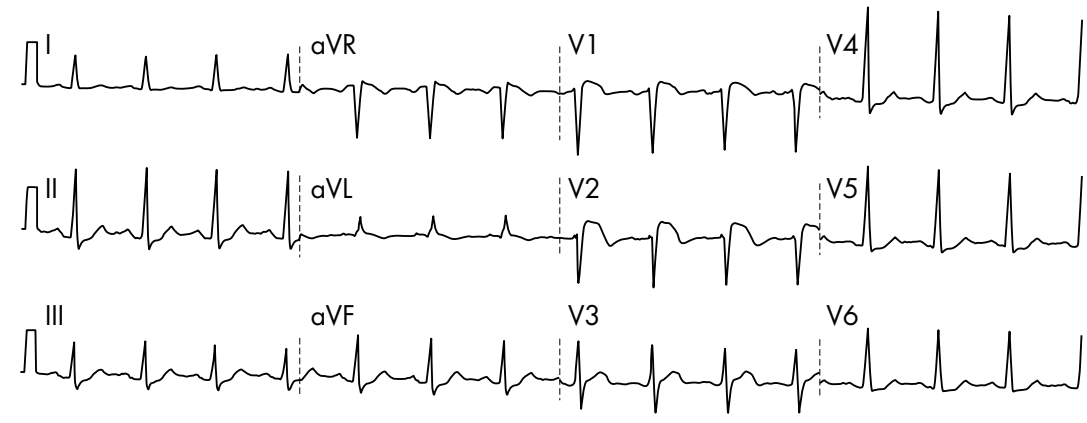

Figure 2 ECG showing classical Brugada changes.

M K Sinha, D Dasgupta, J P Lyons East Surrey Hospital, Redhill, Surrey, UK

Correspondence to: Dr Manas K Sinha, Department of Cardiology, Atkinson Morley Wing, St George's Hospital, Blackshaw Road, London SW17 OQT, UK; msinha@sghms.ac.uk

\section{REFERENCES}

1 Brugada P, Brugada J. Right bundle branch block, persistent ST segment elevation and sudden cardiac death: a distinct clinical and electrocardiographic syndrome. J Am Coll Cardiol 1992;20:1391-6.

2 Brugada R, Brugada J, Antzelevich C, et al. Sodium channel blockers identify risk for sudden death in patients with ST-segment elevation and right bundle branch block but structurally normal hearts. Circulation 2000;101:510-15. 